\title{
Evaluation of Effectiveness of Cleaning of Root Canals using Protaper and K3 Rotary Systems: A SEM Study
}

\author{
${ }^{1}$ Anukriti Tyagi, ${ }^{2}$ BS Keshava Prasad, ${ }^{3} \mathrm{~K}$ Shashikala
}

\begin{abstract}
The aim of this in vitro study was to compare the debris and smear layer removal following root canal preparation using two different rotary systems with scanning electron microscope (SEM). The rotary systems used were Protaper and K3. Forty single rooted permanent mandibular premolars were chosen for the study. They were assigned two groups on the basis of instrumentation used.

The teeth were sectioned at the level of cementoenamel junction and instrumented with Protaper in group $A$ and with $\mathrm{K} 3$ in group $\mathrm{B}$. The root canals were thoroughly irrigated with $5 \mathrm{ml}$ of $2.5 \% \mathrm{NaOCl}$ during instrumentation. After instrumentation, $5 \mathrm{ml}$ of normal saline was used as a final rinse.

The teeth were split longitudinally and the specimens were prepared for SEM evaluation. Scanning electron microscope photomicrographs showed presence of debris and smear layer. The SEM photomicrographs were scored, based on the standard score rating system, and the scores were tabulated accordingly.

The scores obtained from the specimens were subjected to statistical analysis. Results showed opening of dentinal tubules and effective removal of smear layer in group $A$ (Protaper) and no significant difference between both the groups (groups $A$ and $\mathrm{B}$ ) regarding debris.
\end{abstract}

Keywords: Debris, K3, Protaper, SEM, Smear layer.

How to cite this article: Tyagi A, Prasad BSK, Shashikala K. Evaluation of Effectiveness of Cleaning of Root Canals using Protaper and K3 Rotary Systems: A SEM Study. World J Dent 2015;6(1):20-25.

Source of support: Nil

Conflict of interest: None

\section{INTRODUCTION}

Endodontic therapy is essentially a debridement procedure that requires the removal of the irritants of the canal and the periapical tissues if success is to be gained. The debridement may be carried out in various ways and may include instrumentation of the canal, placement of

\footnotetext{
${ }^{1}$ Postgraduate Student, ${ }^{2}$ Professor, ${ }^{3}$ Professor and Head

${ }^{1-3}$ Department of Conservative Dentistry and Endodontics DAPMRV Dental College, Bengaluru, Karnataka, India

Corresponding Author: BS Keshava Prasad, Professor, Department of Conservative Dentistry and Endodontics, DAPMRV Dental College, \#CA 37, 24th Main, JP Nagar I Phase, Bengaluru-560078 Karnataka, India, Phone: 9448505151, e-mail: keshavprasad72@ gmail.com
}

medicaments and irrigants, successful root canal treatment depends mainly on removal of microorganism by proper chemomechanical instrumentation of the canals, thus, enabling removal of both organic tissues by dissolution and inorganic tissues by shaping. Hence, for a successful outcome of root canal treatment. ${ }^{1}$

Cleaning and shaping can be accomplished both with manual and automated root canal instrumentation. Since, most hand root canal preparations are time consuming and technically demanding, much attention has been directed toward automated root canal preparation techniques and especially nickel-titanium rotary instruments. ${ }^{2}$ The introduction of NiTi rotary instruments during last decade has provided better, faster and easier cleaning ability of the root canals. These instruments are made of alloy which has unique mechanical properties, innovative design for crown down preparation. ${ }^{3}$

Rotary NiTi root canal instruments have now become an integral part of the endodontic armamentarium. The use of NiTi alloy in endodontics has allowed the creation of the new instruments. Entirely hand-piece driven systems have appeared, based on the use of rotating and variably tapered instruments. Rotary endodontic instruments fabricated from NiTi alloys have shown to be helpful adjuncts for root canal preparation. ${ }^{4}$

The endodontic instrument must ensure removal of pulp debris from the root canals to render it free from bacteria. The cutting segment of these instruments can be induced to stresses due to insufficient removal of debris. The manner of use and the method of preparation also determine the complete removal of debris along with a proper irrigation protocol. Therefore, these advanced instruments have some characteristics like noncutting tips, varied tapers, radial lands and a variety of crosssections which aids in smear layer removal which is formed during the canal preparation and manipulation of the surface elements of the dentinal walls of the canal. The removal of debris and smear layer from the root canal system prior to obturation is the primary aim of the endodontic treatment. The smear layer is a surface film, approximately 1 to $2 \mathrm{~mm}$ in thickness and combination of organic and inorganic particles on the canal walls after canal preparation and appears as an amorphous irregular layer under scanning electron microscope (SEM) whether a smear layer needs to be removed or retained before canal obturation remains a controversy. Presence 
of smear layer contributes to and it is also a source of irritants for microorganisms. ${ }^{5}$ Whereas, removal of smear layer from the root canal and dentinal tubules provides a thorough disinfection and better adaptation between root canal sealers and the root canal walls. ${ }^{6}$

The ability of rotary instruments to remove dentin and pulpal debris during shaping is connected to flute and cross-sectional design. Protaper universal and K3 are recently introduced NiTi files possessing different configuration as well as design. Both have positive rake angle and noncutting tip design. ${ }^{7}$

Protaper is a new generation of NiTi instruments developed by Dentsply. The cross-sectional design of Protaper is similar to that of a reamer possessing three cutting edges with a convex core without any radial lands. On the other hand, K3 (SybronEndo) is a new generation NiTi rotary file, developed to fulfil and shaping requirements, has triple and asymmetric radial lands of unequal width and depth that aid in penetrating the file from screwing into the canal. They are designed to cut dentin into the canal. They are designed to cut dentin quickly, effectively and safely with unparalleled removal. ${ }^{8}$

Effectiveness of different instruments is based on the variety of ways of evaluating canal debridement. The ability of an endodontic treatment to remove debris from the canals can be assessed with a use of SEM which has proven to be a valuable method for comparing the instruments and techniques.,10

Thereby, comparative evaluation of the debris and smear layer removal following root canal preparation using Protaper universal and K3 rotary system with SEM is carried out.

\section{METHODOLOGY}

A total of 40 single rooted human permanent mandibular premolars extracted for orthodontic and periodontal reasons were selected. The extracted teeth were obtained from Department of Oral and Maxillofacial Surgery, DA Pandu Memorial RV Dental College.

The extracted teeth were cleaned and stored in distilled water. The crowns of the teeth were sectioned at the level of cementoenamel junction. The working length was obtained by placing a 10 size K-file $1 \mathrm{~mm}$ short of the apical foramen. Teeth were then divided into two groups of 20 teeth each. The canals were thoroughly irrigated with $5 \mathrm{ml}$ of $2.5 \% \mathrm{NaOCl}$ solution during instrumentation. After instrumentation, $5 \mathrm{ml}$ of normal saline was used with a plastic syringe as a final rinse.

\section{Instrumentation}

Both types of instruments, i.e. Protaper and K3, were set in permanent rotation with a 4:1 reduction gear anthogyr handpiece at $300 \mathrm{rpm}$.

\section{Group A}

Protaper instrument was used in crown down manner. After complete instrumentation and irrigation, canals were dried with paper points.

\section{Group B}

K3 instrument (G-pack) was used in crown down manner. After canals were shaped and cleaned under frequent and abundant irrigation, a groove was given longitudinally on the roots to split it without penetration of the root canals. The teeth were then carefully split with a hammer and chisel.

\section{SEM Analysis}

Scanning electron microscope provides great depth of field and resolution, thereby providing magnified image of the specimen showing details not visible with light microscope. Therefore, the specimens were air dried at $37^{\circ} \mathrm{C}$ over 24 hours, attached to the coded stubs, sputter coated with $10 \%$ gold-palladium and viewed in SEM. The photomicrographs of the coronal, middle and apical third of the root canals were seen at $1000 \times$ magnification and then each field was graded and calculated from 1 to 5 according to following scoring systems for each region of the root for instruments separately.

\section{Scoring System}

The criteria for smear layer scoring were as follows:

- Score 1: Patent orifices of dentinal tubules.

- Score 2: Few open dentinal tubules with some amount of smear layer.

- Score 3: Some open dentinal tubules with homogenous smear layer present along the entire root canal.

- Score 4: Absence of open dentinal tubules with homogenous smear layer along the entire canal walls.

- Score 5: The root canal walls were covered entirely with thick homogenous smear layer.

The criteria for debris scoring were as follows:

- Score 1: No debris present.

- Score 2: Slight debris.

- Score 3: Debris covering less than $50 \%$ of the sample surface.

- Score 4: More than 50\% of the sample surface covered.

- Score 5: Complete or nearly complete sample surface covered with debris.

\section{STATISTICAL ANALYSIS}

The scores were then statistically analyzed with nonparametric test, i.e. Mann-Whitney test, at a significance level of $\mathrm{p}<0.05$. 
The teeth were sectioned longitudinally and the samples were prepared for SEM study. The photomicrographs were subjected to magnification of 200x and 1000x. The photomicrographs obtained were evaluated using standard scoring system and the score were subjected to statistical analysis.

The instrumented canal walls from both the groups appeared smooth and exhibited varying amounts of remaining debris and smear layer along the entire length of the root canal. The mean scores of debris and smear layer recorded at coronal, middle and apical thirds are shown in Tables 1 and 2 respectively. However, completely cleaned root canals were not observed in any group:

- Null hypothesis: There is no significant difference between the median scores of $\mathrm{K} 3$ and Protaper, i.e. $\eta_{1}=\eta_{2}$.

- Alternate hypothesis: There is a significant difference between the median scores of K3 and Protaper, i.e. $\eta_{1} \neq \eta_{2}$.

- Level of significance: $\alpha=0.05$.

- Statistical technique used: Mann-Whitney test.

- Decision criterion: The decision criterion is to reject the null hypothesis if the p-value is less than 0.05 . Otherwise, we accept the null hypothesis.

\section{Debris}

Higher scores of debris are observed in K3 compared to Protaper at coronal site and the difference in median scores is found to be statistically significant $(\mathrm{p}<0.05)$. K3 has a higher mean and median score of debris compared to Protaper at middle site also. The median difference in debris scores between them is found to be statistically significant $(p<0.05)$. The mean debris score at apical site is found to be higher in K3 compared to Protaper. The median debris scores between them is equal, but the difference in median debris scores between them is found to be statistically significant ( $p>0.05$ ) (Figs 1 and 2).

\section{Smear Layer}

Higher scores of smear layer are observed in K3 compared to Protaper at coronal site and the difference is found to be statistically significant $(\mathrm{p}<0.001)$. Also, in the middle third, $\mathrm{K} 3$ has a higher mean and median score of smear layer compared to Protaper. The difference in smear layer scores between them is found to be statistically significant $(\mathrm{p}<0.001)$. The mean and median smear layer score at apical site is found to be higher in K3 compared to Protaper and the difference in median smear layer scores between them is found to be statistically significant $(\mathrm{p}<$ 0.01) (Figs 3 and 4).

Table 1: Debris score analysis

\begin{tabular}{|c|c|c|c|c|c|c|c|c|}
\hline \multirow[b]{2}{*}{ Site } & \multicolumn{3}{|c|}{ K3 } & \multicolumn{3}{|c|}{ Protaper } & \multirow[b]{2}{*}{ z-value } & \multirow[b]{2}{*}{$p$-value } \\
\hline & Mean & $S D$ & Median & Mean & $S D$ & Median & & \\
\hline Coronal & 2.40 & 0.50 & 2.00 & 1.95 & 0.60 & 2.00 & -2.336 & $0.020^{*}$ \\
\hline Middle & 2.80 & 0.77 & 3.00 & 2.25 & 0.55 & 2.00 & -2.282 & $0.023^{*}$ \\
\hline Apical & 2.80 & 0.70 & 3.00 & 3.00 & 0.73 & 3.00 & -0.885 & 0.429 \\
\hline
\end{tabular}

*Significant difference

Table 2: Smear layer analysis

\begin{tabular}{|c|c|c|c|c|c|c|c|c|}
\hline \multirow[b]{2}{*}{ Site } & \multicolumn{3}{|c|}{ K3 } & \multicolumn{3}{|c|}{ Protaper } & \multirow[b]{2}{*}{ z-value } & \multirow[b]{2}{*}{$p$-value } \\
\hline & Mean & $S D$ & Median & Mean & $S D$ & Median & & \\
\hline Coronal & 3.40 & 0.60 & 3.00 & 2.35 & 0.49 & 2.00 & -4.395 & $<0.001^{*}$ \\
\hline Middle & 3.30 & 0.80 & 3.00 & 2.10 & 0.85 & 2.00 & -3.874 & $<0.001^{*}$ \\
\hline Apical & 4.55 & 0.51 & 5.00 & 3.75 & 0.72 & 4.00 & -3.386 & $<0.001^{*}$ \\
\hline
\end{tabular}

*Significant difference

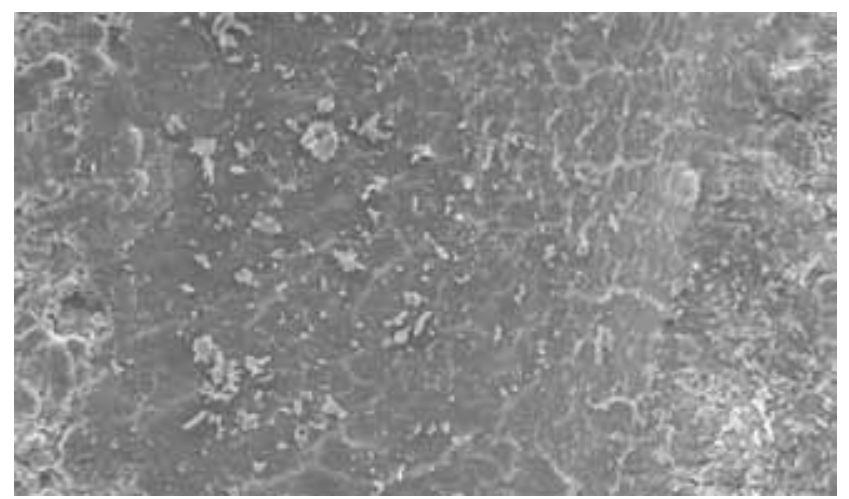

Fig. 1: Scanning electron microscope photomicrograph of debris at apical third: Group A-moderate amount of debris seen covering less than $50 \%$ of the root canal wall

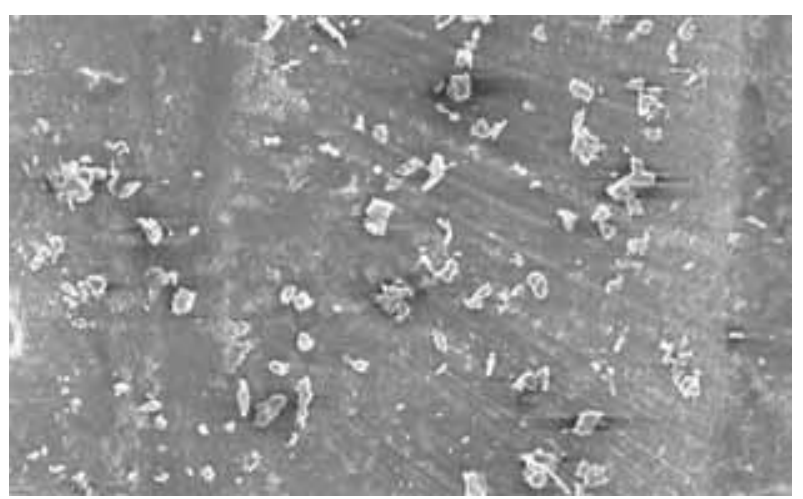

Fig. 2: Scanning electron microscope photomicrograph of debris at apical third: Group B-moderate amount of debris seen covering less than $50 \%$ of the root canal wall 


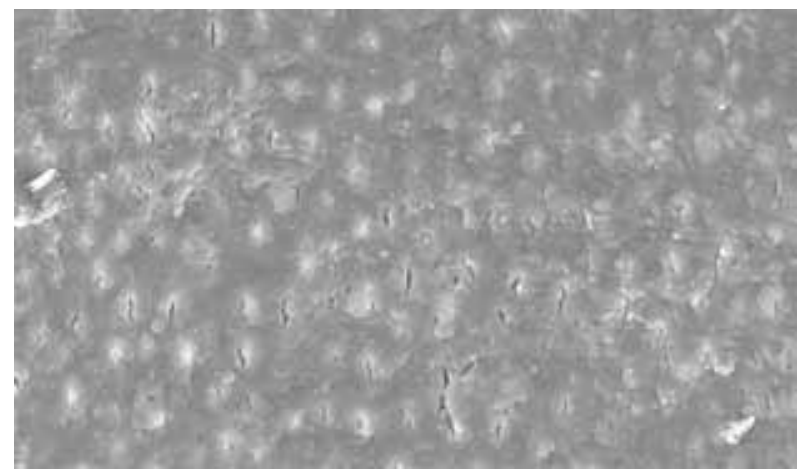

Fig. 3: Scanning electron microscope photomicrograph of smear layer at apical third: Group A-entire root canal covered with smear layer and no open dentinal tubules

\section{DISCUSSION}

A successful root canal treatment requires elimination of bacteria from the root canal system, enlargement of the canal and an impermeable seal to avoid infection or reinfection of the periapical tissues. Thorough debridement of the root canal is considered to be the most important step in endodontic therapy. The main objective of root canal preparation is the removal of the vital/necrotic pulp tissue, remaining necrotic debris and infected dentin, so that the bulk of microorganisms present in the canal will be eliminated. ${ }^{1}$

In this study, the efficiency of K3 and Protaper rotary instruments was assessed using two criteria, i.e. debris and smear layer. Debris is dentinal chips with residual pulp which may be infected in most cases and the pulp tissue which is attached to the canal walls may be vital or necrotic. This debris prevents complete removal of microorganism and also interferes with complete obturation as it occupies a part of the root canal space. ${ }^{1}$

American Association of Endodontists stated that the thickness of the smear layer is about 1 to $2 \mathrm{~mm}$. It is mainly inorganic in nature and produced during canal instrumentation. The role of smear layer in success of an endodontic treatment is remained controversial till date. Due to its deleterious effect the removal of smear layer is still considered desirable. For example, the smear layer contains dentin particles, residual vital or necrotic pulp tissue, protein agglomerates, and blood cells and might harbor microorganisms. Thus, the smear layer might block the openings of the dentinal tubules and in this way impede penetration or diffusion of irrigants or antibacterial medicaments into the dentinal tubules. The complete sealing ability of the canal space may be compromised by the smear layer. The inorganic and organic debris can be effectively removed with use of an antibacterial irrigant along with chelating agents. In this study, $2.5 \%$ concentration of sodium hypochlorite was used as an irrigant. Sodium hypochlorite has both

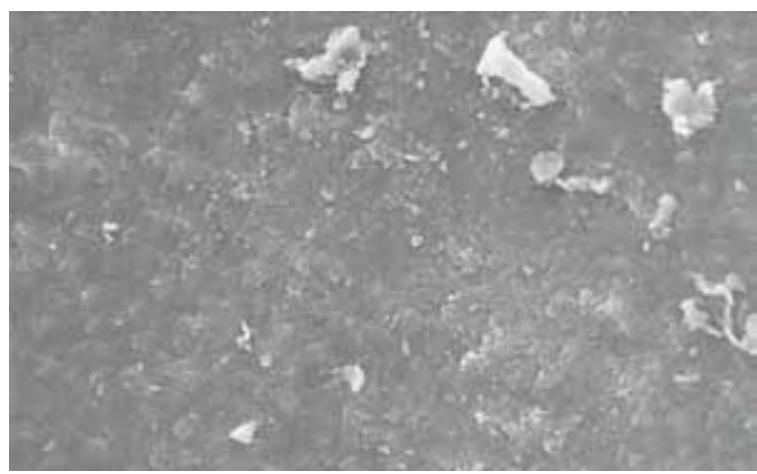

Fig. 4: Scanning electron microscope photomicrograph of smear layer at apical third: Group B-a thick homogenous smear layer covering the entire root canal wall seen with no open dentinal tubules

antibacterial and organic tissue dissolving potential, but it is not capable of removing smear layer completely. The main objective of this study was to compare cleaning ability of two instrumentation techniques under similar conditions. Hence, a simple irrigation protocol was used to avoid associations of various irrigating solutions.

The instruments used in the present study were Protaper and K3 rotary instruments. Protaper system was introduced in 2000 by Dentsply Maillefer, Switzerland. It consists of three shaping and three finishing files with a multiple and progressively changing tapers ranging from 2 to $8 \%$. The cross-section is convex triangular with no radial lands and positive rake angle..$^{15}$ On the contrary, K3 file system was designed by Dr John McSpadden (SybronEndo) and has an asymmetrical cross-section with three radial lands and positive rake angle. ${ }^{12}$

Scanning electron microscope has been used to evaluate the cleanliness of canal walls after endodontic instrumentation as high resolution and magnification images are obtained with SEM techniques. The quantative measurements of coronal, middle and apical sites were taken randomly. Studies have been based on examination of canal walls at magnifications varying from $50 \times$ to 5000x. In this study, 200x magnification was employed for scoring debris as it offered a wider view and allowed the detection of large fragments of dentinal debris at low magnification. Whereas, $1500 \times$ magnification was used for scoring the smear layer as high magnification covered too small a surface and gave accurate information, also it allows the identification of the smear layer and the evaluation of the aperture of the tubule openings. ${ }^{11}$ The study has used a more quantitative approach by attributing a score to each view of a micrograph.

This study examined the cleaning efficacy of K3 and Protaper instruments on the basis of a numerical evaluation method of smear layer and debris with SEM evaluation at coronal, middle and apical portions of the root canals. ${ }^{5}$ Two-by-two comparison of the instruments 
in the present study demonstrated that with none of the systems used in the study could an acceptable cleanliness of the root canal walls was obtained.

During the past few years, NiTi rotary instruments with advanced blade designs have been developed to improve the efficacy during root canal preparation. As observed in this study, in terms of debris, the coronal third of the canals prepared using K3 and Protaper showed less debris with a score of two and with no significant difference between the two groups. Whereas, at the middle third of the root canal difference in the score was seen. K3 showed a higher score of three as compared to Protaper. In the apical third, no difference in the debris score was seen and both the instruments showed higher score of three.

Various morphologic designs affect the cleaning property of rotary instruments. It has been reported that the instrumental design can play an important role in the canal cleanliness. Debris removal can be affected by the flute design of the instrument. ${ }^{13}$ Variable helix angles and pitch are other features that can improve the removal of the debris formed by the instrumentation. Since, Protaper has a variable helical angle, it results in efficient debris removal compared to $\mathrm{K} 3$ which has consistent helical angle which allows debris to accumulate in the coronal part of the file, blocking the escape way of the debris. ${ }^{5}$

For smear layer, Protaper showed cleaner canal walls at all the three levels as compared to the canals prepared with K3. The coronal and middle portions of the root canal prepared using Protaper were cleaner when compared to $\mathrm{K} 3$ and resulted in few open dentinal tubules. Whereas, in the apical third of the canal the scores were high for both groups, with K3 showing higher scores than that of Protaper. A homogenous covering of the smear layer was observed in the entire root canal in apical third with no open dentinal walls.

The smear layer removal depends on the file design. Presence of radial lands and rake angle affects the smear layer removal. Radial lands of the instruments perform a planning action rather than a cutting action on the root canal walls, thereby decreasing the cleaning ability. In the study, K3 rotary files consists of three radial lands, whereas Protaper has no radial land. Therefore, K3 presented with inferior cleaning ability, showing incomplete removal of the smear layer. Also, presence of positive rake angle improves the smear layer removal efficacy. Both the systems used in the study have positive rake angles and thereby good cutting efficacy. Also, the triangular cross-sectional design of Protaper system makes it superior to $\mathrm{K} 3$.
The results on the cleaning ability underline the limited efficacy of endodontic instruments for cleaning the root canal at apical third. Additional irrigation played crucial role to provide sufficient disinfection of the endodontic system. ${ }^{14}$

In this study, Protaper rotary instruments resulted in significantly less debris and smear layer compared to K3 rotary system. Significant differences were found between the coronal and the apical third regarding debris and the smear layer. It can be concluded that Protaper instrument's design is found to be effective in reducing the amount of debris and smear layer. Therefore, the rotary instrumentation using Protaper yields cleaner canals as compared to $\mathrm{K} 3$ rotary instruments.

\section{CONCLUSION}

Within the limitations of this study can be concluded that, disparate instrumental design of rotary nickeltitanium files influenced the cleanliness of the root canal, Protaper rotary instrument used resulted in better smear layer removal as compared to K3 rotary instrument and, therefore, resulting in more open dentinal tubules in the coronal and middle third as compared to apical third. No statistically significant difference was found between both the systems regarding debris removal. Debris was effectively removed in coronal third but middle and apical third did not show any significant difference and were covered with debris.

\section{REFERENCES}

1. Schafer E, Vlassis M. Comparative investigation of two rotary nickel-titanium instruments: Protaper versus RaCe. Part 2. Cleaning effectiveness and shaping ability in severely curved root canals of extracted teeth. Int Endod J 2004;37(4):239-248.

2. Gambarini G, Laszkiewicz J. A scanning electron microscopic study of debris and smear layer remaining following use of GT rotary instruments. Int Endod J 2002;35(5):422-427.

3. Gianluca $\mathrm{G}$. The $\mathrm{K} 3$ rotary nickel titanium instrument system. Endodontic Topics 2005;10(1):179-182.

4. Rodig T, Hulsmann M, Kahlmeier C. Comparison of root canal preparation with two rotary NiTi instruments: Profile .04 and GT rotary. Int Endod J 2007;40(7):553-562.

5. Zand Vahid, Bidar M, Ghaziani Pari, Rahimi Saeed, Shahi S. A comparative SEM investigation of the smear layer following preparation of root canals using nickel titanium rotary and hand instruments. J Oral Sci 2007;49(1);47-52.

6. Torabinejad M, Handysides. Clinical implications of the smear layer in endodontics: a review. Oral Surg Oral Med Oral Pathol Oral Radiol Endod 2002;94(6):658-66.

7. Foschi F, Nucci C, Marchionni L. SEM evaluation of canal wall dentine following use of Mtwo and Protaper NiTi rotary instruments. Int Endod J 2004;37(12):832-839.

8. Elham A, Reiko EW, Ebrahim AK, Debris SH. Retention and wear in three different nickel-titanium rotary instruments. Aust Endod J 2006;32(3):107-111. 
9. Saulius D, Balciuniene I. A scanning electron microscopic study of debris and smear layer remaining following use of AET instruments and K-flexofiles. Stomatologija Baltic Dental and Maxillofacial Journal 2006;8(3):70-75.

10. Saulius D, Balciuniene I. A scanning electron microscopic study of debris and smear layer remaining following use of AET and Protaper instruments. Acta Medica Lituanica 2006;13(4):249-257.

11. Bertrand MF, Pizzardini P, Muller M, Medioni E, Rocca JP. The removal of smear layer using the Quantec system. A study using the scanning electron microscope. Int Endod J 1999;32:217-224.
12. Mounce Richard. The K3 rotary nickel-titanium file system. Dent Clin N Am 2004;48:137-157.

13. Almannai Ghanim. The efficacy of different rotary instrumentation techniques on canal cleanliness: A comparative electron microscope study. Karolinska Institutet. 2004.

14. Paque F, Musch U, Hulsmann M. Comparison of root canal preparation using $\mathrm{RaCe}$ and Protaper rotary Ni-Ti instruments. Int Endod J 2005;38:8-16.

15. Vaudt J, Bitter K, Kielbassa AM. Evaluation of rotary root canal instruments in vitro: a review. Endodontology 2007; 1(3):189-203. 